\title{
The Effect of Bracing on Spinopelvic Rotation and Psychosocial Parameters in Adolescents with Idiopathic Scoliosis
}

\author{
Yalda Khoshhal ${ }^{1}$, Maryam Jalali ${ }^{1}$, Taher Babaee ${ }^{1}$, Hassan Ghandhari ${ }^{2}$, Jeffrey L. Gum ${ }^{3}$ \\ ${ }^{1}$ Department of Orthotics and Prosthetics, School of Rehabilitation Sciences, Iran University of Medical sciences, Tehran, Iran \\ ${ }^{2}$ Bone and Joint Reconstruction Research Center, Shafa Orthopedic Teaching Hospital, Iran University of Medical Sciences, Tehran, Iran \\ ${ }^{3}$ Department of Orthopedic Surgery, Norton Leatherman Spine Center, Louisville, KY, USA
}

Study Design: Retrospective study.

Purpose: To evaluate the effect of bracing on spinopelvic rotation and psychosocial parameters in adolescents with idiopathic scoliosis (AIS).

Overview of Literature: Complex three-dimensional deformity in AIS is proposed to influence the spinopelvic parameters and psychosocial condition in adolescents; however, few studies have quantitatively evaluated these parameters.

Methods: Thirty AIS who were prescribed a brace were included in the study. The patients' standing postero-anterior and total spine radiographs were used to measure the primary curve Cobb angle, vertebral rotation, and pelvic rotation. Apical vertebral rotation (AVR), upper AVR, and lower AVR were measured using the Nash-Moe method. Pelvic rotation was determined using the left-to-right hemipelvic width ratio. The curve pattern was classified as per the Lenke classification system. In all, 14 patients had a type I curve, five had type II, six had type III, one had type IV, and four had type V curves. Brace compliance was subjectively evaluated by interviewing the patients and their parents. The health-related quality of life (HROOL) and stress level of the recruited patients were assessed using the Brace Questionnaire and Bad Sobernheim Stress Questionnaire, respectively.

Results: The Cobb angle significantly decreased with at least 6 months of brace use. AVR correction changed significantly; however, no such results were observed for upper and lower AVR. Pelvic rotation and psychosocial parameters were not significantly affected by brace use. No statistically significant correlation was observed between brace compliance and curve correction.

Conclusions: The Cobb angle and AVR are crucial measurements that help evaluate the treatment efficacy in AIS with small curves who undergo brace treatment. HROOL and pelvic axial rotation are not influenced by the brace treatment.

Keywords: Scoliosis; Brace; Stress; Pelvis; Vertebra; Rotation

\section{Introduction}

Adolescent idiopathic scoliosis (AIS), with a prevalence of
$0.5 \%-5.2 \%$ in adolescents aged $10-16$ years old, is defined as a lateral curvature of the spine combined with axial vertebral rotation and/or sagittal plane imbalance [1].

Received Dec 1, 2018; Revised Mar 2, 2019; Accepted Mar 19, 2019

Corresponding author: Maryam Jalali

Department of Orthotics and Prosthetics, School of Rehabilitation Sciences, Iran University of Medical Sciences, Madadkaran Avenue, Shahnazari St., Madar Square, Mirdamad Blvd., Tehran, Iran

Tel: +98-21-2222-0947, Fax: +98-21-2222-0946, E-mail: jalali.m@iums.ac.ir 
Bracing is the most effective non-operative treatment for immature adolescents with progressive scoliosis curves. The results of a multicenter randomized controlled trial (BrAIST: bracing in adolescent idiopathic scoliosis trial) showed that bracing had a positive influence on the natural course of AIS in terms of halting the curve progression in skeletally immature patients [2].

With respect to non-surgical treatment, frontal plane deformity has been the primary outcome broadly used by clinicians or researchers, and less attention has been paid to rotational components of the deformity [2,3]. Dubousset et al. [4] proposed the theory that the pelvis behaves as a vertebra; therefore, it is likely that scoliosis deformity involves the pelvis. Gum et al. [5] reported that pelvic rotations occurred mostly toward the convexity of thoracic curves and described the rotations as compensatory movements of the pelvis. Pelvic rotation has mostly been mentioned as an independent factor in previous studies and has been measured pre- and postoperatively $[5,6]$. Moreover, few studies have quantitatively evaluated the effect of bracing on axial vertebral rotation in AIS $[3,7,8]$. Yamane et al. [3] found that in patients with insufficient vertebral rotation control, the rate of bracing failure increased. The pelvis was not included in the Yamane study; however, as per the pelvic-vertebra theory that considers the pelvis as a vertebra $[9,10]$, the behavior of the pelvis should be considered.

Psychosocial domains, such as stress, self-image, mental health, functional activity, and depression are also affected by scoliosis [11,12]. Treatment success in scoliosis depends not only on the radiological assessments, but is also correlated with the patient's quality of life [13]. Recent studies have shown that the evaluation of healthrelated quality of life (HRQOL) parameters in AIS is as important as radiological measurements [13,14]. Different questionnaires have been used to evaluate quality of life domains; however, among them, the only brace-oriented instrument is the Brace Questionnaire (BrQ) [15]. AIS may experience stress at baseline, and the brace treatment may increase the stress level. The Bad Sobernheim Stress Questionnaire (BSSQ) has also been developed as a twopart series of short questions to evaluate the stress level in AIS [16].

The current study was designed to evaluate the effect of bracing on less-dealt-with aspects of scoliosis, i.e., axial vertebrae and pelvic rotation and to determine the correlation between psychosocial parameters in AIS. We hypothesized that (1) bracing could affect the rotational parameters caused by scoliosis, and that (2) a positive correlation exists between the domains of the BrQ and BSSQ and curve correction.

\section{Materials and Methods}

This study was approved by the Human Research Ethics Committee of the Iran University of Medical Sciences (case no., 1396.9411502003). Written informed consent was obtained from all the patients and their parents before they were enrolled in the study.

\section{Participants}

From June 2017 to March 2018, the clinical records of all AIS who visited the rehabilitation centers in Tehran, Iran as outpatients for their routine follow-up were retrospectively reviewed. Of these, 146 patients fulfilled the inclusion criteria. Of those who met the criteria, 30 AIS (28 girls and two boys), with a mean age of 13 years agreed to participate in the study. The inclusion criteria of the study were as follows: age $>10$ years at the initiation of brace treatment, Risser sign $\leq 2$, an initial Cobb angle of $20^{\circ}-45^{\circ}$, wearing of the brace for at least 6 months [13] and for at least 12 hours a day [2], and no previous treatment. Patients with congenital malformation of the spine, neuromuscular scoliosis, or other musculoskeletal disorders were excluded.

\section{Radiological assessments}

Out-of-brace postero-anterior, total spine, and standing radiographs were obtained to measure the parameters of interest, including (1) the primary curve Cobb angle, (2) vertebral rotation, and (3) pelvic rotation.

The following three vertebral rotations for three vertebral levels was measured using the Nash-Moe method [17]: apical vertebral rotation (AVR), upper AVR, and lower AVR. The Nash-Moe method divides the vertebra width into six equal parts, and the grade of rotation was determined by the location of the convex side pedicle (Fig. 1). In the absence of displacement, the vertebra rotation grade is considered zero.

Pelvic rotation was indirectly calculated as the left-toright hemi-pelvic width ratio [5]. The anterior-superior iliac spine, as a lateral landmark, and the ilium in the 


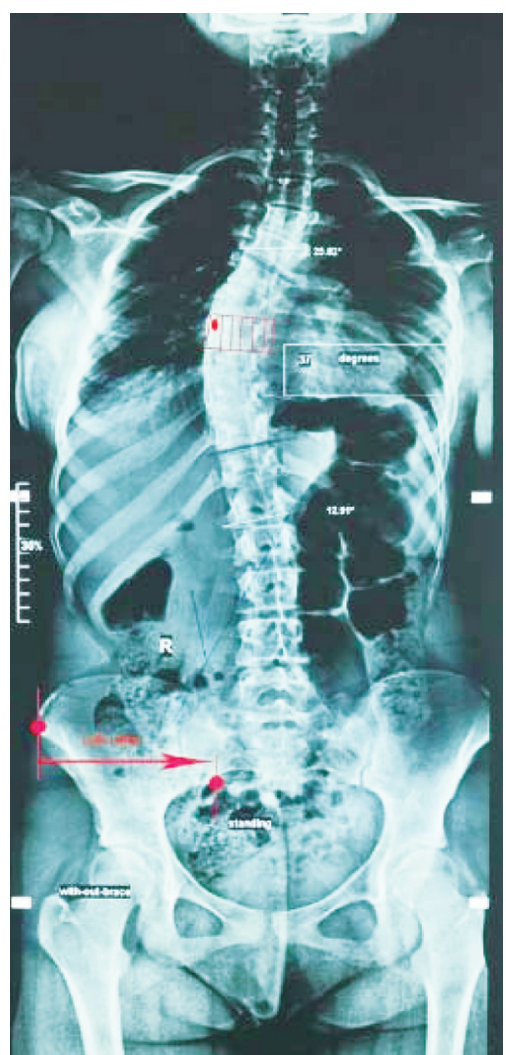

Fig. 1. Illustration of the hemi-pelvic landmarks (inferior ilium at the sacroiliac joint, medially, and anterior-superior iliac spine, laterally) and vertebral rotation measurements.

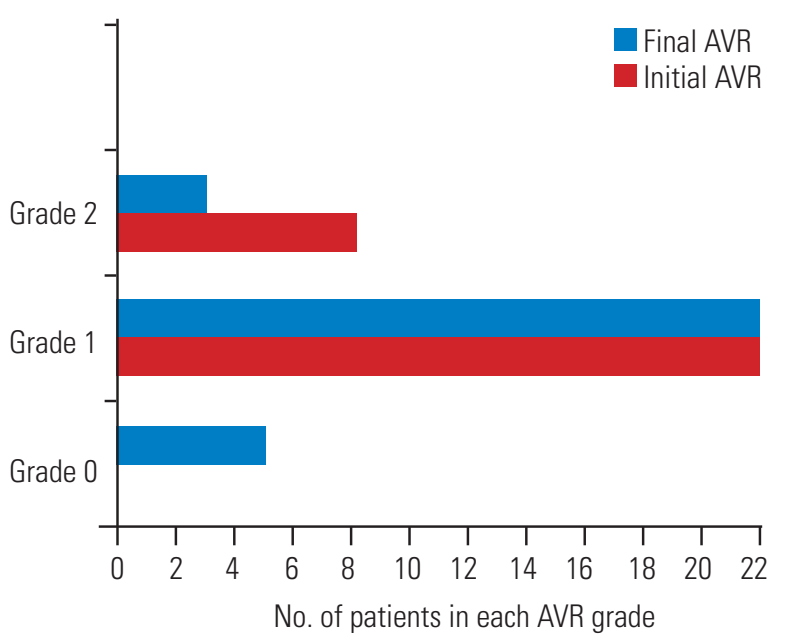

Fig. 2. The number of patients in each AVR grade before (initial) and after (final) orthotic treatment. AVR, apical vertebral rotation.

sacroiliac joint, as a medial landmark, were marked. The perpendicular linear distance of the two landmarks was measured on each side (Fig. 2).

Intra- and inter-rater reliability of this method was 0.97 and 0.88 , respectively, as reported by Gum [5]. When the left-to-right hemi-pelvic width ratio was $<1$, the pelvis rotated toward the right, and when this ratio was $>1$, the pelvis rotated toward the left.

The Cobb angle was measured using the patients' posterior standing radiographs. These parameters were measured from the out-of-brace baseline radiographs and the patients' last available radiographs. All the measurements were performed by two independent raters (Y.K. and T.B.), and a meeting between these two raters was held to establish a consensus in case of any discrepancies.

The curve pattern was categorized as per the Lenke classification system [7]; no special criteria existed in terms of the curve pattern.

\section{Clinical assessments}

The Beighton scoring system was used to obtain patients' ligamentous laxity [18]. This scoring system comprises five simple clinical maneuvers described below and a measuring scale ranging from 0 to 9: (1) passive dorsiflexion of the fifth finger is $>90^{\circ}$; (2) the thumb shows passive opposition to the volar aspect of the forearm; (3) elbow hyperextension is $>10^{\circ}$; (4) knee hyperextension is $>10^{\circ}$; and (5) patients place hands flat on the floor without bending the knees.

Maneuvers 1-4 are completed for both the sides; each positive test gives 1 point. A total score of $\geq 4$ is considered to indicate joint hyper laxity. Moreover, the patient's height and weight were measured by an examiner to calculate body mass index.

\section{Brace compliance assessments}

Brace compliance was subjectively assessed by asking the patients and their parents the following two questions: (1) Have you used the brace as prescribed? (2) For how many hours daily are you using the brace? [14]. The answers were recorded as number of hours per day.

\section{Health-related quality of life assessments}

HRQOL and stress levels of the patients were evaluated using the Persian versions of the Bad Sobernheim Stress Questionnaire (P-BSSQ) and the Brace Questionnaire (P-BrQ) $[19,20]$, respectively. Patients were asked to complete these questionnaires at the final follow-up. The 
P-BSSQ assesses the stress level caused by the treatment or the deformity. This questionnaire comprises eight questions about the patient's feelings about the deformity and treatment. The patient can score 1-4 for each question, as described in the guideline introduced by the questionnaire's developers. Higher scores represent lower stress attributable to scoliosis or the brace treatment. The P-BrQ comprises 34 questions in eight domains. The total score ranges from 20 to 100 . Higher scores are considered to represent better quality of life.

\section{Statistical analyses}

The data were analyzed using IBM SPSS statistical software ver. 22.0 (IBM Corp., Armonk, NY, USA). Assumption of the normality of the data was evaluated using the Shapiro-Wilk test. All the parameters had normal distribution except brace compliance and vertebral rotation. In order to compare the mean values of the baseline and the final Cobb angles, a paired sample $t$-test was performed. The Wilcoxon signed-rank test was used to compare the median values of the baseline and the final AVR, upper AVR, and lower AVR. The Spearman and Pearson correlation analyses were conducted to establish the relationships between the stress level and subscales of the P-BrQ, radiologic parameters, and clinical parameters of the patients. All $p$-values $<0.05$ were considered to indicate a statistically significant difference. All the values are reported as mean and standard deviation.

\section{Results}

\section{Patient characteristics}

Total 30 patients fulfilled the inclusion criteria of the study. The mean age of the recruited patients at the initial bracing was $13.1 \pm 1.7$ years, and the mean initial Cobb angle was $30.6^{\circ} \pm 10.3^{\circ}$. As per the Lenke classification system, the patients' curves were categorized into the following five types [21]: type I or main thoracic $(n=14)$, type II or double thoracic $(n=5)$, double thoracic and lumbar curves or type III $(n=6)$, type IV or thoracolumbar $(n=1)$, and type $V$ or lumbar $(n=4)$. In this study, one patient had TLSO, and 29 patients had the Milwaukee brace. The mean brace-wearing time was $22.4 \pm 12$ months, and patients wore the braces for a mean of $19.8 \pm 3.9$ hours every day. The body mass index of the included patients was $18.6 \pm 1.8 \mathrm{~kg} / \mathrm{m}^{2}$. The average score on the Beighton test was $7 \pm 1$, demonstrating generalized ligamentous laxity.

\section{Comparison of the radiological parameters}

The mean differences between the pre-treatment and post-treatment Cobb angles were statistically significant $(p<0.001)$ as per the paired sample $t$-test analysis (Table 1$)$. The Cobb angle was increased in three patients after wearing the brace; for two patients, no difference was noted. Pelvic rotation was negligible at both, the initial bracing and at final follow-up ( $p>0.05)$.

The initial AVR in $27 \%$ of the patients was grade 2; for

Table 1. Mean values and paired sample $t$-test comparison measurement results for the Cobb angle and pelvic rotation before and after the treatment

\begin{tabular}{lccc} 
Characteristic & Pre-treatment & Post-treatment & $p$-value \\
Cobb angle $\left(^{\circ}\right)$ & $30.6 \pm 10.6$ & $23.8 \pm 9.9$ & 0.00 \\
Pelvic rotation & $1.00 \pm 0.1$ & $0.97 \pm 0.1$ & 0.35 \\
\hline
\end{tabular}

Values are presented as mean \pm standard deviation or number (\%). All $p$-values $<0.05$ were considered statistically significant.

Table 2. Wilcoxon signed-rank test results for the comparison of vertebral rotation grades before and after the treatment

\begin{tabular}{lccc} 
Characteristic & Pre-treatment & Post-treatment & $p$-value \\
AVR & 2 & 1 & 0.004 \\
Upper AVR & 1 & 1 & 0.083 \\
Lower AVR & 1 & 1 & 0.071 \\
\hline
\end{tabular}

All $p$-values $<0.05$ were considered statistically significant.

AVR, apical vertebral rotation. 


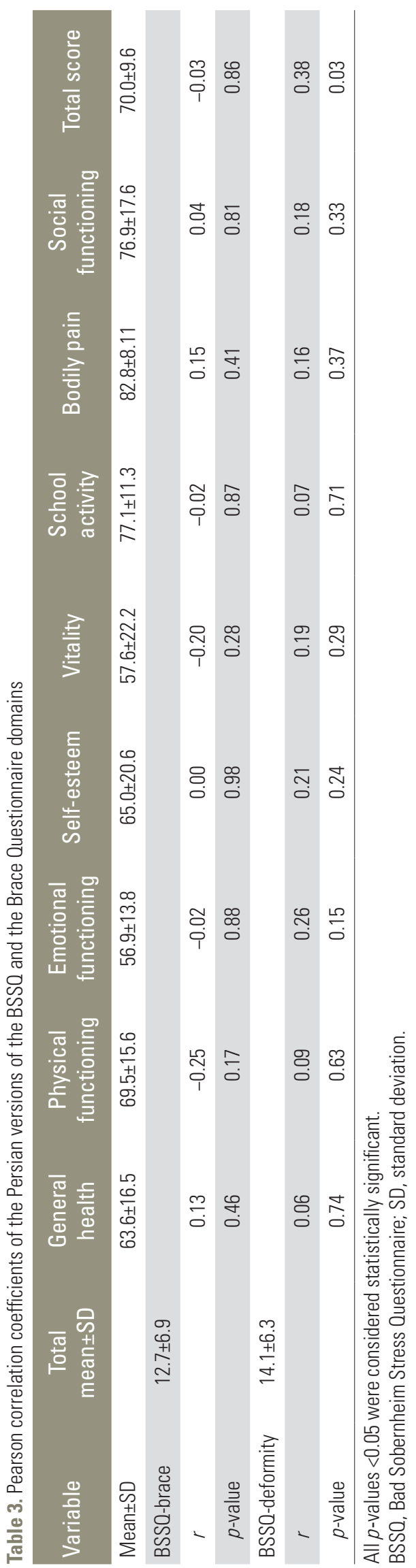

the other patients, the AVR was grade 1. Neutral apical vertebra (grade 0 ) was not mentioned at the initial bracing; however, after treatment, it was noted in $17 \%$ of the patients (Fig. 2). From the initiation of brace treatment to the final follow-up, AVR correction was statistically significant $(p<0.05)$; however, no such result was obtained for upper and lower AVR (Table 2). Curve correction, as measured by the Cobb angle, was better achieved in patients with more reduction in AVR $(p<0.05)$; however, curve correction was not significantly correlated with pelvic rotation correction $(p>0.05)$.

\section{Correlations between the subscales and the total score of the Persian versions of the Bad Sobernheim Stress Questionnaire and the Brace Questionnaire}

The mean scores of the P-BrQ and the P-BSSQ were $12.7 \pm 6.9$ and $14.1 \pm 6.3$, respectively. The mean total score of the P-BrQ was 70.0 \pm 9.6 . Patients with lower deformityrelated stress appeared to have a higher quality of life $(p<0.05)$ (Table 3). Patients with more reduction in curve severity were less stressed about their braces $(p<0.05$, $r=0.37$ ) (Table 4). No statistically significant correlation existed among ligamentous laxity, brace compliance, body mass index, and curve correction.

\section{Discussion}

Bracing is the most effective non-operative treatment for AIS, with a great focus on radiologic findings in the coronal plane parameters $[2,22,23]$. One of the prognostic factors for brace effectiveness is in-brace curve correction [13], a parameter that was statistically significant in the current study. Vertebral rotation plays a role in AIS pathogenesis and disregarding it is believed to be related with brace failure [3]. In the present study, in-brace AVR was significantly reduced; however, no statistically significant changes were observed for upper and lower apical vertebrae. The apical vertebra is the most frontally translated vertebra, and brace pads are designed and placed based on the apical vertebra; therefore, antero-medial directed pad loading of the brace is mostly applied to this region and achieves higher correction. The results also showed that the AVR was further reduced in patients with better curve correction. These findings are consistent with the findings of Yamane et al. [3] that showed that rotational correction plays a greater role in the effectiveness of bracing than 
Table 4. Pearson correlation coefficients of curve correction, P-BrO, and P-BSSO

\begin{tabular}{lccc} 
Degrees of curve correction & P-BrO total score & BSSO-brace & BSSO-deformity \\
$r$ & 0.18 & 0.37 & 0.32 \\
$p$-value & 0.32 & 0.04 & 0.08 \\
\hline
\end{tabular}

All $p$-values $<0.05$ were considered statistically significant.

P-BrO, the Persian version of the Brace Questionnaire; P-BSSQ, the Persian version of the Bad Sobernheim Stress Questionnaire; BSSQ, Bad Sobernheim Stress Questionnaire.

Cobb angle correction.

Sagittal and coronal pelvic parameters (pelvic incidence, sacral slope, and pelvic tilt) are now being considered in AIS and their treatment protocols [24,25]; however, axial plane rotations of the pelvis require further research. In the current study, pelvic axial rotation was comparable before and after the treatment. To clarify this finding, we should mention that the initial Cobb angle of the patients was $30.6^{\circ}$. A previous study by Gum et al. [5] showed a statistically significant degree of pelvic rotation for severe curves, with a mean Cobb angle of $61^{\circ}$. Therefore, pelvic rotation for minor curves that undergo brace treatment was not statistically significant at pre-treatment, and including this parameter in-brace treatment protocols may not be as necessary as the inclusion of vertebral rotation. In the current study, most patients had main thoracic curves. However, the lumbar curves may behave differently.

In the current study, HRQOL was not statistically influenced by brace treatment. This may be due to the patients' small curve magnitudes at initial bracing. This finding is supported by previous studies that showed that bracing did not impact the patient's psychological well-being. These studies also showed that patients with moderate curves who underwent brace treatment had the same HRQOL score as the healthy control group [26,27]. Participants in the current study had moderate levels of stress; therefore, the lack of a significant effect on their HRQOL was acceptable because a positive correlation between the total score of the P-BrQ and deformity-related stress demonstrates higher HRQOL resulting from lower stress levels due to the deformity. Another finding of the study was a correlation between the Cobb angle correction and brace-related stress. With continued decrease in the Cobb angle, the patient will find the brace to be more effective and become less stressed about the outcome of bracing.

The outcome of bracing is also influenced by factors, such as joint laxity, body mass index, and brace compliance $[10,14]$. The current study showed no significant correlation between the above parameters and Cobb angle correction. This may be attributable to assessment methods that could be under- or over-estimated. Brace compliance was determined subjectively, and joint laxity was measured with a clinical Beighton test.

To our knowledge, no study has considered vertebrae and pelvic rotational parameters at the same time during brace treatment in AIS. However, this study has certain limitations. First, the lumbar flexibility was not quantitatively examined using side-bending radiographs during the study. In patients with higher lumbar flexibility, the body mostly tends to compensate for the thoracic curve in the lumbar area, resulting in less rotation in the pelvis for the maintenance of body balance, as reported by Wang et al. [28]. Second, brace compliance can be more accurately measured when registered objectively. The sagittal orientation of the facet joints in lumbar area block axial rotation; therefore, the rotational component of scoliosis in this region may cause more pelvic rotation. Further research is warranted to investigate the pelvic rotation parameters in patients who use braces with main lumbar and thoracolumbar curves

\section{Conclusions}

In-brace Cobb angle and AVR degree significantly decreased in participants after at least 6 months of brace wear. Pelvic axial rotation was negligible before and after orthotic treatment assessments. Curve correction, as measured with the Cobb angle, was better achieved in patients with more reduction in AVR. Deformity did not induce stress in the participants, and the HRQOL was not significantly influenced by brace treatment. Thus, apex vertebral rotation deserves more attention in orthotic treatment of AIS. 


\section{Conflict of Interest}

No potential conflict of interest relevant to this article was reported.

\section{Acknowledgments}

We would especially like to thank Zahra Hedayati from the Rehabilitation Center of the Iranian Red Crescent Society for her support when we recruited patients and collected data for this research.

\section{Author Contributions}

Conception and design: TB; acquisition of data: YK; analysis and interpretation of data: YK, TB, HG; drafting the article: YK, MJ, TB, HG, JLG; critically revising the article: YK, MJ, TB, HG, JLG; reviewed submitted version of manuscript: MJ, TB, JLG; and approved the final version of the manuscript on behalf of all authors and study supervision: MJ, TB.

\section{References}

1. Konieczny MR, Senyurt H, Krauspe R. Epidemiology of adolescent idiopathic scoliosis. J Child Orthop 2013;7:3-9.

2. Weinstein SL, Dolan LA, Wright JG, Dobbs MB. Effects of bracing in adolescents with idiopathic scoliosis. N Engl J Med 2013;369:1512-21.

3. Yamane K, Takigawa T, Tanaka M, Sugimoto Y, Arataki S, Ozaki T. Impact of rotation correction after brace treatment on prognosis in adolescent idiopathic scoliosis. Asian Spine J 2016;10:893-900.

4. Dubousset J, Charpak G, Dorion I, et al. A new 2D and $3 \mathrm{D}$ imaging approach to musculoskeletal physiology and pathology with low-dose radiation and the standing position: the EOS system. Bull Acad Natl Med 2005;189:287-97.

5. Gum JL, Asher MA, Burton DC, Lai SM, Lambart LM. Transverse plane pelvic rotation in adolescent idiopathic scoliosis: primary or compensatory? Eur Spine J 2007;16:1579-86.

6. Lucas B, Asher M, McIff T, Lark R, Burton D. Estimation of transverse plane pelvic rotation using a posterior-anterior radiograph. Spine (Phila Pa 1976) 2005;30:E20-7.
7. Hwang CJ, Lee CS, Lee DH, Cho JH. Progression of trunk imbalance in adolescent idiopathic scoliosis with a thoracolumbar/lumbar curve: is it predictable at the initial visit? J Neurosurg Pediatr 2017;20:450-5.

8. Katz DE, Durrani AA. Factors that influence outcome in bracing large curves in patients with adolescent idiopathic scoliosis. Spine (Phila Pa 1976) 2001;26:2354-61.

9. Duval-Beaupere G, Schmidt C, Cosson P. A Barycentremetric study of the sagittal shape of spine and pelvis: the conditions required for an economic standing position. Ann Biomed Eng 1992;20:451-62.

10. Khodaei M, Pacheco-Pereira C, Trac S, Chan A, Le LH, Lou E. Radiographic methods to estimate surgical outcomes based on spinal flexibility assessment in patients who have adolescent idiopathic scoliosis: a systematic review. Spine J 2018;18:2128-39.

11. Aulisa AG, Guzzanti V, Perisano C, et al. Determination of quality of life in adolescents with idiopathic scoliosis subjected to conservative treatment. Scoliosis 2010;5:21.

12. Gallant JN, Morgan CD, Stoklosa JB, Gannon SR, Shannon CN, Bonfield CM. Psychosocial difficulties in adolescent idiopathic scoliosis: body image, eating behaviors, and mood disorders. World Neurosurg 2018;116:421-32.

13. Chan SL, Cheung KM, Luk KD, Wong KW, Wong MS. A correlation study between in-brace correction, compliance to spinal orthosis and health-related quality of life of patients with adolescent idiopathic scoliosis. Scoliosis 2014;9:1.

14. Brox JI, Lange JE, Gunderson RB, Steen H. Good brace compliance reduced curve progression and surgical rates in patients with idiopathic scoliosis. Eur Spine J 2012;21:1957-63.

15. Vasiliadis E, Grivas TB, Gkoltsiou K. Development and preliminary validation of Brace Questionnaire $(\mathrm{BrQ})$ : a new instrument for measuring quality of life of brace treated scoliotics. Scoliosis 2006;1:7.

16. Botens-Helmus C, Klein R, Stephan C. The reliability of the Bad Sobernheim Stress Questionnaire (BSSQbrace) in adolescents with scoliosis during brace treatment. Scoliosis 2006;1:22.

17. Nash CL Jr, Moe JH. A study of vertebral rotation. J Bone Joint Surg Am 1969;51:223-9.

18. Czaprowski D, Kotwicki T, Pawłowska P, Stolinski L. Joint hypermobility in children with idiopathic 
scoliosis: SOSORT award 2011 winner. Scoliosis 2011;6:22.

19. Rezaei Motlagh F, Pejam H, Babaee T, Saeedi H, Hedayati Z, Kamali M. Persian adaptation of the Bad Sobernheim stress questionnaire for adolescent with idiopathic scoliosis. Disabil Rehabil 2018:1-5.

20. Rezaee S, Jalali M, Babaee T, Kamali M. Reliability and concurrent validity of a culturally adapted Persian version of the brace questionnaire in adolescents with idiopathic scoliosis. Spine Deform 2019;7:553-8.

21. Lenke LG, Betz RR, Harms J, et al. Adolescent idiopathic scoliosis: a new classification to determine extent of spinal arthrodesis. J Bone Joint Surg Am 2001;83:1169-81.

22. Dolan LA, Weinstein SL. Surgical rates after observation and bracing for adolescent idiopathic scoliosis: an evidence-based review. Spine (Phila $\mathrm{Pa} 1976$ ) 2007;32(19 Suppl):S91-100.

23. Katz DE, Herring JA, Browne RH, Kelly DM, Birch JG. Brace wear control of curve progression in adolescent idiopathic scoliosis. J Bone Joint Surg Am 2010;92:1343-52.
24. Moon ES, Nanda A, Park JO, et al. Pelvic obliquity in neuromuscular scoliosis: radiologic comparative results of single-stage posterior versus two-stage anterior and posterior approach. Spine (Phila Pa 1976) 2011;36:146-52.

25. Chen RQ, Hosogane N, Watanabe K, et al. Reliability analysis of spino-pelvic parameters in adult spinal deformity: a comparison of whole spine and pelvic radiographs. Spine (Phila Pa 1976) 2016;41:320-7.

26. Cheung KM, Cheng EY, Chan SC, Yeung KW, Luk $\mathrm{KD}$. Outcome assessment of bracing in adolescent idiopathic scoliosis by the use of the SRS-22 questionnaire. Int Orthop 2007;31:507-11.

27. Schwieger T, Campo S, Weinstein SL, Dolan LA, Ashida S, Steuber KR. Body image and quality of life and brace wear adherence in females with adolescent idiopathic scoliosis. J Pediatr Orthop 2017;37:e51923.

28. Wang ZW, Wang WJ, Sun MH, et al. Characteristics of the pelvic axial rotation in adolescent idiopathic scoliosis: a comparison between major thoracic curve and major thoracolumbar/lumbar curve. Spine J 2014;14:1873-8. 